\title{
Mesospheric dust and its secondary effects as observed by the ESPRIT payload
}

\author{
O. Havnes ${ }^{1}$, L. H. Surdal ${ }^{2}$, and C. R. Philbrick ${ }^{3, *}$ \\ ${ }^{1}$ Department of Physics and Technology, University of Troms $\varnothing$, Troms $\varnothing$, Norway \\ ${ }^{2}$ Narvik University College, Narvik, and Andøya Rocket Range, Andenes, Norway \\ ${ }^{3}$ Pennsylvania State University, Electrical Engineering Department, USA \\ *now at: Physics Department and Marine, Earth and Atmospheric Science Department, North Carolina State University, \\ Raleigh NC, USA
}

Received: 14 July 2008 - Revised: 18 December 2008 - Accepted: 7 January 2009 - Published: 5 March 2009

\begin{abstract}
The dust detector on the ESPRIT rocket detected two extended dust/aerosol layers during the launch on 1 July 2006. The lower layer at height $\sim 81.5-83 \mathrm{~km}$ coincided with a strong NLC and PMSE layer. The maximum dust charge density was $\sim-3.5 \times 10^{9} \mathrm{e} \mathrm{m}^{-3}$ and the dust layer was characterized by a few strong dust layers where the dust charge density at the upper edges changed by factors 2-3 over a distance of $\lesssim 10 \mathrm{~m}$, while the same change at their lower edges were much more gradual. The upper edge of this layer is also sharp, with a change in the probe current from zero to $I_{\mathrm{DC}}=-10^{-11} \mathrm{~A}$ over $\sim 10 \mathrm{~m}$, while the same change at the low edge occurs over $\sim 500 \mathrm{~m}$. The second dust layer at $\sim 85-92 \mathrm{~km}$ was in the height range of a comparatively weak PMSE layer and the maximum dust charge density was $\sim-10^{8} \mathrm{e} \mathrm{m}^{-3}$. This demonstrates that PMSE can be formed even if the ratio of the dust charge density to the electron density $P=N_{d} Z_{d} / n_{e} \lesssim 0.01$.

In spite of the dust detector being constructed to reduce possible secondary charging effects from dust impacts, it was found that they were clearly present during the passage through both layers. The measured secondary charging effects confirm recent results that dust in the NLC and PMSE layers can be very effective in producing secondary charges with up to $\sim 50$ to 100 electron charges being rubbed off by one impacting large dust particle, if the impact angle is $\theta_{i} \gtrsim 20-35^{\circ}$. This again lends support to the suggested model for NLC and PMSE dust particles (Havnes and Næsheim, 2007) as a loosely bound water-ice clump interspersed with a considerable number of sub-nanometer-sized meteoric smoke particles, possibly also contaminated with meteoric atomic species.
\end{abstract}

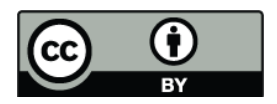

Correspondence to: O. Havnes (ove.havnes@uit.no)
Keywords. Atmospheric composition and structure (Aerosols and particles; Middle atmosphere - composition and chemistry; Instruments and techniques)

\section{Introduction}

During the last decades, considerable attention has been focused on the observation and understanding of the role of mesospheric dust particles, or aerosols. We will, in the following, call these dust particles although they, for the most part, probably consist of water ice. They were first recognized as being present as visual particles in the noctilucent clouds (NLC) (Gadsden and Schröder, 1989; Thomas, 1991) and later it was suspected that non-visual small dust particles could cause the so-called electron bite-outs, strong local depletions of the electron density which often are measured by rocket probes in the summer mesosphere (Pedersen et al., 1969; Ulwick et al., 1988; Havnes et al., 1996). Lidar observations of NLC particles (von Cossart et al., 1999) indicate that visual NLC particles had an average radius of $\sim 50 \mathrm{~nm}$ and average density of $\sim 8 \times 10^{7} \mathrm{~m}^{-3}$ which is confirmed by satellite measurements (Eremenko et al., 2005). The radar PMSE phenomenon (Cho and Röttger, 1997; Ecklund and Balsley, 1981; Rapp and Lübken, 2004) was also suspected earlier as being linked to dust particles, due to its similarity in seasonal variation and height distribution to the NLC. Simultaneous and co-located observations of PMSE and NLC conclude that they most likely have common causes (von Zahn and Bremer, 1999).

Direct in situ observations of the mesospheric dust were attempted by sampling (Hallgren et al., 1973) but the results were largely inconclusive although it was stated that a few large (diam. $\sim 100$ to $700 \mathrm{~nm}$ ) mesospheric particles

Published by Copernicus Publications on behalf of the European Geosciences Union. 


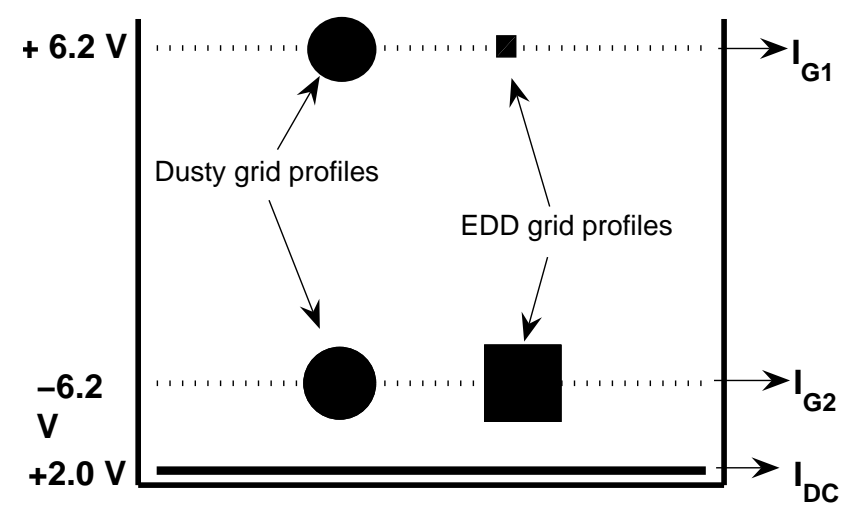

Fig. 1. The principle of the EDD probe, with its upper and lower grids of square profiles and the circular ones for DUSTY. The grid profiles are not shown to scale. The side edges are $1 \mathrm{~mm}$ for G2 and $0.25 \mathrm{~mm}$ for G1. For DUSTY both grids have profiles with diameter of $0.8 \mathrm{~mm}$.

were probably collected. Later observations by rocket mass spectrometers (Björn and Arnold, 1981; Kopp et al., 1985; Schulte and Arnold, 1992) indicated the presence of massive ions, or microclusters. It is, however, unclear how the effect of airflow could have affected these observations (Horanyi et al., 1999; Hedin et al., 2006). The first probe to unambiguously detect heavy charged mesospheric dust particles of sizes probably from a few nm and upwards, was the DUSTY probe flown in 1994 (Havnes et al., 1996). This probe showed that large amounts of negatively charged dust of a charge density of up to $\sim-4 \times 10^{9} \mathrm{e} \mathrm{m}^{-3}$ was present in two strong electron bite-outs. The dust was not observed visually or by lidars but a strong PMSE layer was present. Later, rocket probe observations (Mitchell et al., 2001; Havnes et al., 2001a, b; Smiley et al., 2006) confirm the presence, also of subvisual dust, at NLC and PME conditions.

It is now becoming increasingly clear that secondary charging effects, due to impacting dust particles on rocket payloads and their probes, can affect some measurements (Zadorozhny et al., 1993; Havnes et al., 1996; Vostrikov et al., 1997; Gumbel and Witt, 1998) and probably sometimes totally dominate them (Barjatya and Swenson, 2006; Havnes and Næsheim, 2007). In the last paper, it was demonstrated that secondary charging by dust particles impacting on surfaces at a high impact angle $\theta_{i}$ (= angle with the normal to the surface), which fragmented and carried away negative charge, could give the appearance of incoming positive charges. Similar effects have been observed in laboratory experiments with small ice particles with sizes of the order of $10 \mathrm{~nm}$ impacting on surfaces, with velocities of $\sim 1 \mathrm{~km} / \mathrm{s}$ (e.g. Andersson and Pettersson, 1997; Tomsic, 2001; Gridin et al., 2004). The required effectivity for the secondary charge production of the mesospheric dust is much larger than what is observed for pure water-ice particles in experiments. This, combined with a modelling of the impacts on the dust probe grids as a function of payload spin rotation angle, led Havnes and Næsheim (2007) to conclude that a model for the mesospheric dust could be a fairly loosely bound ice particle in which a considerable number of small meteoric particles (Rosinski and Snow, 1961; Hunten et al., 1980; Megner et al., 2006) of radius $\lesssim 1 \mathrm{~nm}$ are embedded. Upon impact, the large dust particle was assumed to fragment into many small subparticles each containing one or more meteoric smoke particle. While most of the water ice on the fragments would sublimate during the impact, the meteoric smoke particles made of metals and silicate compounds (Plane, 2003) should survive and a considerable fraction of them would carry away a negative charge.

The model apparently requires that dust particles, with a radius of $\sim 50 \mathrm{~nm}$ or more, are capable of carrying away $\sim-50$ e to -100 e after impact and fragmentation. The requirement of a secondary charge production, much higher than for experimentally observed impacts of pure ice particles, must be tested in future dust probe experiments. The present dust experiment had as one of its purposes, to test the reality of the large secondary production required by observations with the dust probe DUSTY (Havnes and Næsheim, 2007).

In Sect. 2, we describe the experiment. In Sect. 3, we will present the basic observations, while in Sect. 4, we will analyse the currents to the probe. In Sect. 5, we find the secondary charge production, the net dust current and the corresponding dust charge density as a function of height.

\section{The dust experiment and the launch conditions}

The ESPRIT Dust Detector (EDD) was one of 13 experiments (6 engineering demonstrations and 7 scientific) on the ESPRIT rocket (Engineering and Scientific Projects for Research and International Teamwork), a joint US and Norwegian student project payload (Philbrick et al., 2007; Edwards et al., 2007). It was launched 06:39 UT on 1 July 2006 from Andøya Rocket Range as number 3 (SPIRIT III) in a series of student rocket payloads.

The EDD dust detector (Fig. 1) is a bucket which is closed for external electrons and ions at the top by a grid (G1) biased at $+6.2 \mathrm{~V}$, with another grid $(\mathrm{G} 2)$ at $-6.2 \mathrm{~V}, 20 \mathrm{~mm}$ above the bottom plate $(\mathrm{DC})$ at $+2.0 \mathrm{~V}$. The inner diameter of the probe was $80 \mathrm{~mm}$. G1 is made of grid wires with a square profile thickness $(0.25 \times 0.25 \mathrm{~mm})$, all in the same plane and with intergrain distances (from centre to centre) of $6.5 \mathrm{~mm}$. G2 has the same shape and intergrain distance but the thickness of the rectangular wires is increased to $1 \mathrm{~mm}$. The currents from G1, G2 and the gold-plated DC are all measured. The EDD probe is similar to the original DUSTY probe (Havnes et al., 1996) but the shape of the grids has been altered to change the production of secondary charges from the impacting dust particles. Also, the G1 wire thickness was reduced from $0.8 \mathrm{~mm}$ on DUSTY to $0.25 \mathrm{~mm}$, resulting in a 
reduction of the ratio of the dust probe opening which is covered, from $\sigma_{1}=0.23$ to 0.08 . On the $\mathrm{G} 2$ the grid wire thickness is increased to $1 \mathrm{~mm}$, and the covered fraction increases from $\sigma_{2}=0.23$ on DUSTY to 0.28 on EDD.

In the following, we will concentrate on the currents $I_{\mathrm{G} 2}$ and $I_{\mathrm{DC}}$ measured on $\mathrm{G} 2$ and DC, as was done in an earlier analysis (Havnes and Næsheim, 2007). Grid 2 and the bottom plate are close together and secondary charge effects on $I_{\mathrm{G} 2}$ will show up, with an opposite sign, in the current $I_{\mathrm{DC}}$. This enables us to find both the secondary current contribution $I_{S}$ to $I_{\mathrm{G} 2}$ (and $-I_{S}$ to $I_{\mathrm{DC}}$ ) and the current due to dust before hitting or passing G2. The possible secondary effects from $\mathrm{G} 1$, with its low effective area of $\sigma_{1}=0.08$, will be neglected. The current to this grid is dominated by the capture of electrons. It appears that this current is severely affected by the sweep frequency of Langmuir probes on booms (Escobar et al., 2007), which are swept at a frequency of $1 \mathrm{~Hz}$ (Fig. 2). The change of the grid profiles from circular to square (see Fig. 1) will reduce the production of secondary charges compared to that of DUSTY, for similar impacts. This is because the area of G2, which now gives impacts at large impact angles $\theta_{i}$ (measured from the normal of the surface to the direction from where the dust comes), will be strongly reduced on EDD compared to DUSTY if the coning angle $\gamma$ (angle between payload axis and velocity direction) of the payload is small to moderate. At $\gamma=0$ there should be no secondary production on the grids of EDD, since dust impacts will only be on the upper side of the square with $\theta_{i}=0^{\circ}$. The circular cross-section of a DUSTY grid will always present surfaces to the incoming dust where $0 \leq \theta_{i} \leq 90$, regardless of $\gamma$, and the secondary production is therefore much more likely than for EDD. The coning of the ESPRIT payload, as measured with a sun sensor, was $\gamma \sim 13^{\circ}$ as the payload passed the NLC and PMSE layers (Reichard et al., 2007). We will assume that the coning is around the velocity direction. This results in that some of the edges of one grid square, which are aligned along the payload axis (Fig. 1), will have dust particle impact angles in the range $\theta_{i}>90-\gamma \sim 77^{\circ}$. Such impacts will lead to secondary charge production (Havnes and Næsheim, 2007).

The ESPRIT payload was launched during NLC and PMSE conditions. The ALOMAR lidars (von Zahn et al., 1995) observed strong NLC from 82.1 to $84.1 \mathrm{~km}$ at the launch time. This NLC layer varied considerably in height with time and extended down to $\sim 81 \mathrm{~km}$ only $\sim 10 \mathrm{~min}$ before launch and well below $81 \mathrm{~km} \sim 45 \mathrm{~min}$ after launch. The NLC layer apparently coincided well with a PMSE layer from $\sim 82.2$ to $84.4 \mathrm{~km}$. This layer, observed with the ALWIN radar (Latteck et al., 1999), was relatively stable in height around the launch time, with some variation in height intensity profile. Well above the lower NLC/PMSE layer there was a second and weaker layer of PMSE (no NLC) from 86.2 to $89.5 \mathrm{~km}$. This layer varied a great deal in height and intensity on time scales of a few minutes around launch time.

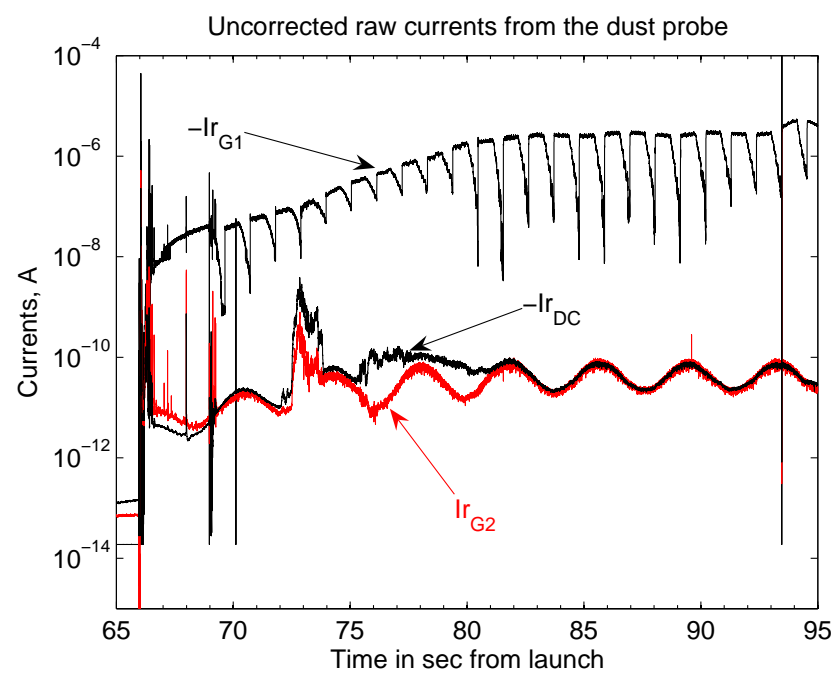

Fig. 2. The raw currents to G1, G2 and the bottom plate. The currents are shown as positive but both $I r_{\mathrm{G} 1}$ and $I r_{\mathrm{DC}}$ were measured as negative currents while $I r_{\mathrm{G} 2}$ is positive.

\section{Observations by the ESPRIT Dust Detector (EDD)}

The currents from the grids G1 and G2 and the bottom plate DC (Fig. 1) were measured by logarithmic electrometers. The absolute values of the raw currents $I r_{\mathrm{G} 1}, I r_{\mathrm{G} 2}$ and $I r_{\mathrm{DC}}$, recorded during the flight in and near to the NLC and PMSE layers, are shown in Fig. 2. We will not, as earlier stated, consider the current to G1 which is strongly affected by a separate Langmuir probe which is being swept at $\sim 1 \mathrm{~Hz}$ (Escobar et al., 2007). Grid 1 is mainly intended to close the interior to the ambient ion and electron plasma and the comparatively large currents to it, is evidently caused by the capture of electrons.

In the uncorrected currents $I r_{\mathrm{G} 2}$ and $I r_{\mathrm{DC}}$, we clearly see that there are impacts of dust in the time span $\sim 72 \mathrm{~s}$ to $74 \mathrm{~s}$ which corresponds to the NLC and lower PMSE layer. The $I_{\mathrm{G} 2}$ is positive, while $I_{\mathrm{DC}}$ is negative. This difference, in the sign of the current, is a signature of that secondary electric charge production is taking place and that it can be dominant in $I_{\mathrm{G} 2}$. This is discussed in more detail in Sects. 4 and 5.

There is a sinusoidal-like background contribution to the currents $I r_{\mathrm{G} 2}$ and $I r_{\mathrm{DC}}$, ranging from about $10^{-11}$ to $10^{-10} \mathrm{~A}$, at the coning period of $T_{c} \sim 3.84 \mathrm{~s}$. We have attempted to remove this unwanted current contribution by the following two procedures. We have fitted a sinusoidal curve, of period $T_{c}$ to the background and expected it to have local maxima at the times 70.5, 74.3, 81.8 and $85.6 \mathrm{~s}$ which we find, from the inspection of $I r_{\mathrm{G} 2}$ and $I r_{\mathrm{DC}}$ in Fig. 2, are in regions where a negligible amount of dust is present. In Fig. 3 we show the raw currents $I r_{\mathrm{G} 2}$ and $I r_{\mathrm{DC}}$, and the background sinusoidal current as a blue line. For $I r_{\mathrm{G} 2}$ we have also plotted the result for the background when we adapted a Fourier-series to the $I r_{\mathrm{G} 2}$ (where the strong signal between 


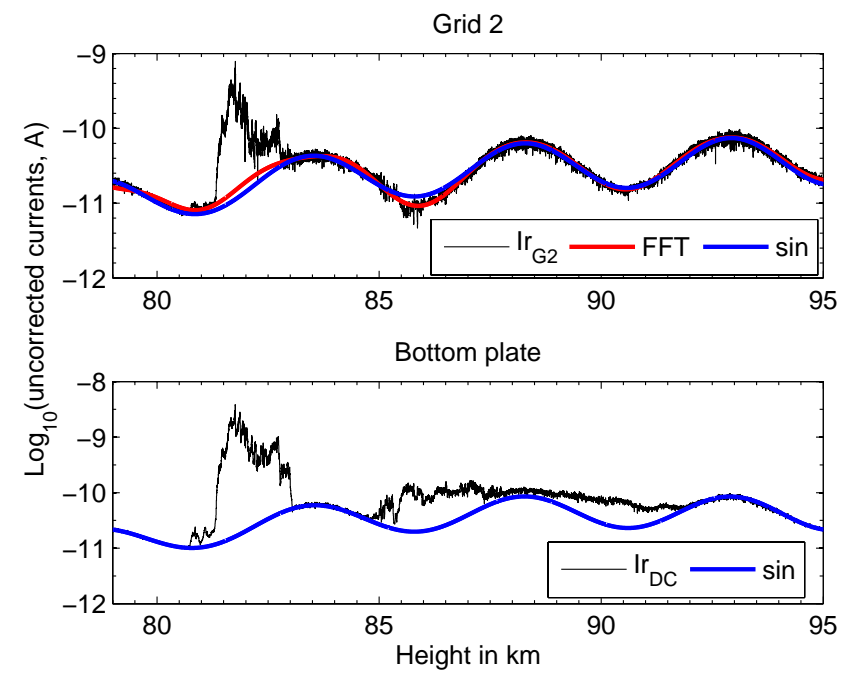

Fig. 3. Showing the raw data from G2 and the bottom plate and the representation of the background contribution by a Fourier series with 12 terms as red, and by a sinus-like curve as a blue curve. $I r_{\mathrm{G} 2}$ is positive and $I r_{\mathrm{DC}}$ is negative.

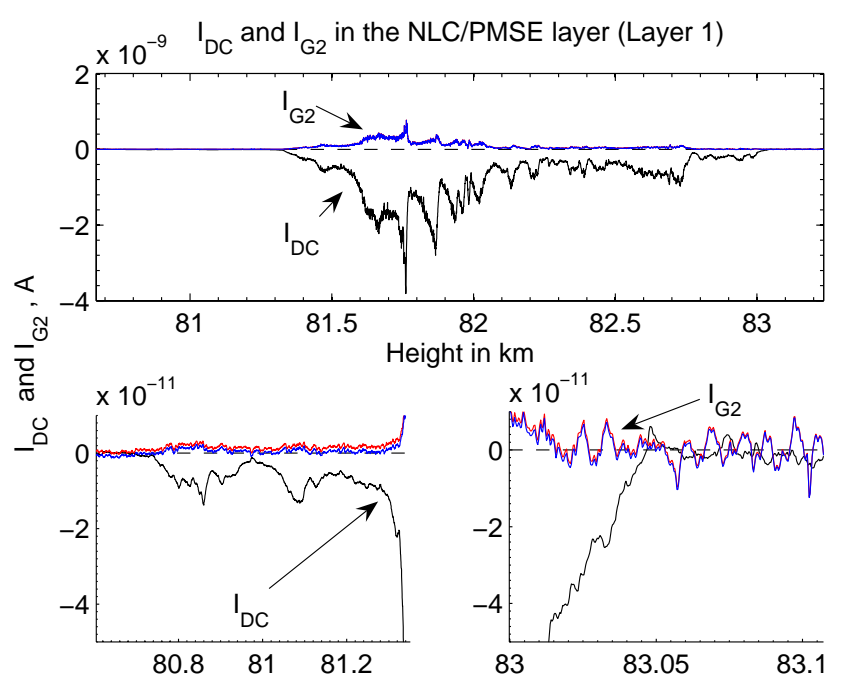

Fig. 4. The corrected $I_{\mathrm{DC}}$ (black curves) and $I_{\mathrm{G} 2}$ with Fourier correction (red curve) and sinus correction (blue curve) for the NLC/PMSE layer.

$t=72.51$ and $73.65 \mathrm{~s}$ was replaced by a straight line) and replotted it (red curve) including only the first 12 terms, which in our case, are terms with periods from $1.7 \mathrm{~s}$ and up to the time interval of the plot of $20 \mathrm{~s}$. Subtracting the background variations from $I r_{\mathrm{G} 2}$ and $I r_{\mathrm{DC}}$ give the net $I_{\mathrm{G} 2}$ and $I_{\mathrm{DC}}$. In Fig. 4 we give the net currents for the lower NLC/PMSE layer, which we will call layer 1 . We have blown up the lower and upper parts of the layer to show details of the edge regions. In Fig. 5, we show the much weaker upper PMSE layer which we will call layer 2 . The maximum currents, shown in Fig. 5 for layer 2, are $\sim 30$ times weaker than in

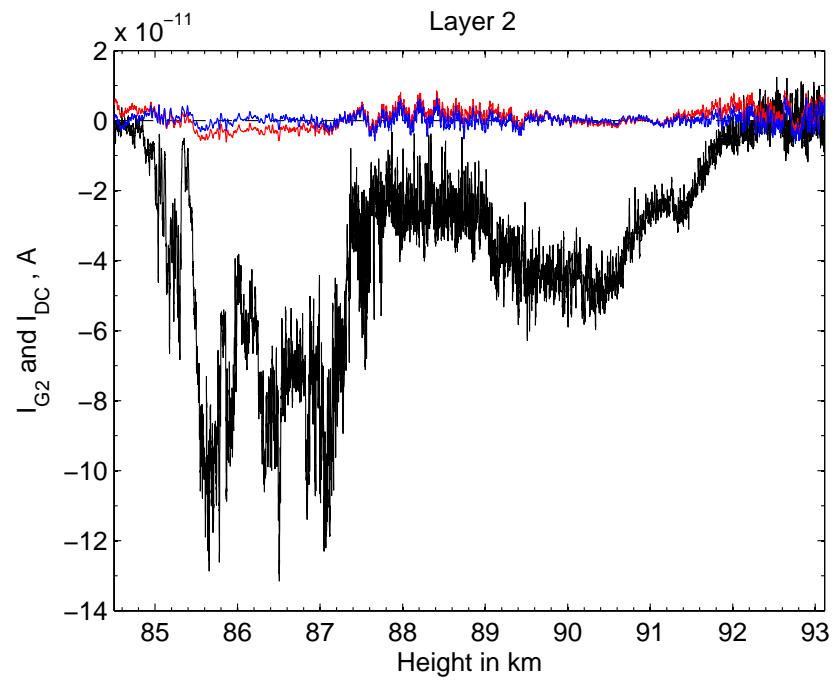

Fig. 5. As for Fig. 4 but now for layer 2, the upper PMSE layer.

layer 1 . The $I_{\mathrm{DC}}$ currents for both layers are strong enough to be relatively slightly affected by uncertainties in the background sinusoidal current except at the edges and in the region from $\sim 87.5$ to $89 \mathrm{~km}$ where there appears to be a gap in the PMSE layer. The $I_{\mathrm{G} 2}$ current is very weak, and correspondingly uncertain, in the whole height range of layer 2 . While it is positive in most or all of layer 1, it goes slightly negative in the height region 85.5 to $86.5 \mathrm{~km}$, while it is very low in the rest of layer 2.

Figure 4a shows that in the NLC/PMSE layer there are a few sublayers, all with sharp upper edges and a slower decline of density in their lower parts. A similar case for the upper and lower edges of the total layer 1, with a decline from a current $\sim-10^{-11} \mathrm{~A}$ to less than $-10^{-12} \mathrm{~A}$ over only $\sim 10 \mathrm{~m}$ at the upper edge and $\sim 500 \mathrm{~m}$ in the lower part of the layer. We have also investigated if the $I r_{\mathrm{DC}}$ current is affected by periodic phenomena related to the rocket, such as the rotation period or the Langmuir sweep period of $\sim 1 \mathrm{~s}$. In Fig. 6, we show the FFT power spectra of the NLC/PMSE (layer 1) in the top panel, the PMSE (layer 2) in the middle panel, and a region above the PMSE layer in the lower panel. The power spectrum has been normalized so that the power at the rotation frequency $f_{R}=5.45 \mathrm{~Hz}$ is put equal to 1 . There are no dominant frequencies in the NLC/PMSE or PMSE layer of panels (a) and (b) although $f_{R}$ and $4 f_{R}$ are weakly present. Above the layers, as shown in panel (a), the $f_{R}$ and $4 f_{R}$ are more clearly apparent but the dominant frequency is the coning frequency at $f_{c}=1 / 3.84$ which is outside the scale of Fig. 6. 


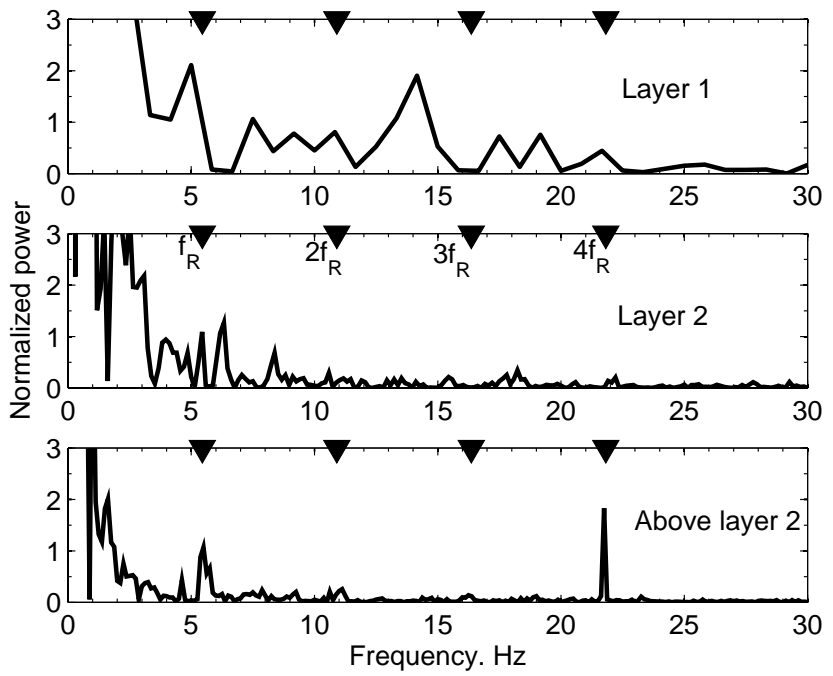

Fig. 6. Fourier frequency analysis of layer 1,2 and the region above the layers. We show the payload spin frequency $f_{r}$ and its harmonics up to $4 f_{R}$.

\section{Analysis of the probe currents to Grid 2 and the bot- tom plate}

The interior of the dust probe is closed to the ambient thermal ions and electrons by grid 1, while the heavy dust particles pass through it (Havnes et al., 1996). Very small particles of radius $\leq 4-5 \mathrm{~nm}$ can be seriously affected by drag from the airflow around the payload (Horanyi et al., 1999; Rapp et al., 2005; Hedin et al., 2007) and may be prevented from reaching the interior of the dust probe. We will assume that the fraction of dust charge density carried by the very smallest dust particles, which are not detected by the dust probe, is small compared to the total dust charge density. Model calculations show that in the lower PMSE layer, dust of radius down to around $3 \mathrm{~nm}$ will probably enter the probe with an efficiency of around 0.7, while for the upper layer slightly smaller dust should also enter the probe with a high efficiency (Hedin et al., 2007). The comparatively small size limits detection, together with recent findings that the majority of dust particles smaller than $2-3 \mathrm{~nm}$ probably are neutral at sunlit conditions (Havnes and Kassa, 2009) due to the effect of photodetachment (Weingartner and Draine, 2001) support our assumption. The dust will impact on G2 and the bottom plate and lead to currents

$I_{\mathrm{G} 2}=\sigma_{2} I_{D}+I_{S}$

$I_{\mathrm{DC}}=\left(1-\sigma_{2}\right) I_{D}-I_{S}$

Here $I_{D}$ is the current inside the probe between grid 1 and 2 , while $\sigma_{2}=0.28$ is the ratio of the area of grid 2 to that of the probe opening. The current $I_{D}$ is related to the total dust charge density $N_{d} Z_{d} e$ in the dust layers by

$I_{D}=\left(1-\sigma_{1}\right) N_{d} Z_{d} e \cdot V_{R} \cdot \pi R_{p}^{2} \cdot \cos \gamma$
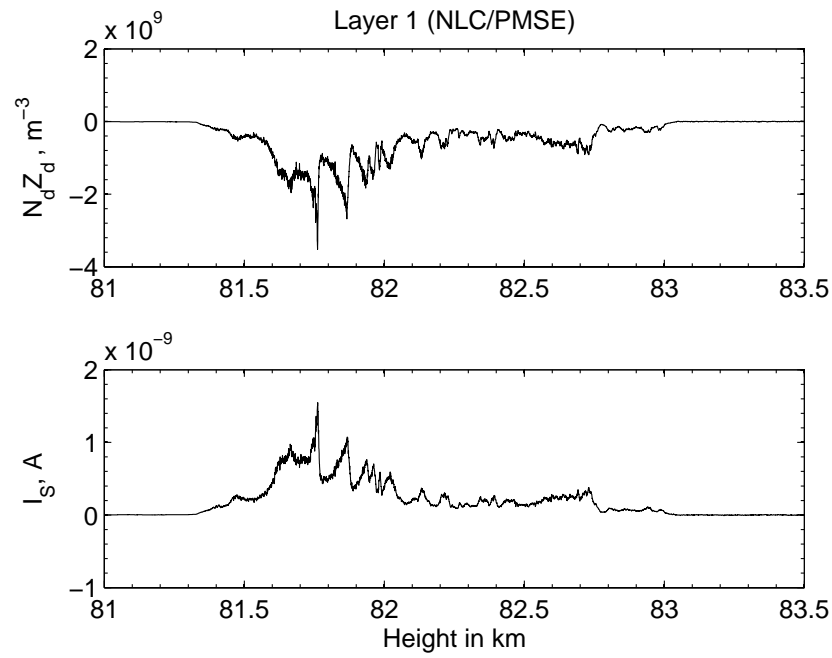

Fig. 7. The dust charge number density and the secondary current as a function of height in layer 1 (NLC/PMSE).

$N_{d} Z_{d} e$ symbolizes the total dust charge density given by an integration over all dust sizes of the dust size distribution multiplied with the dust charges as a function of size. $N_{d}$ is the dust density, $Z_{d}$ the dust charge number and $e=1.602 \times 10^{-19} \mathrm{C}$. The rocket velocity is $V_{R}$, the dust probe radius is $R_{p}$ and $\gamma$ is the coning angle. A fraction $\sigma_{1}=0.08$ of the dust charge flux into the probe is removed by grid 1 . We disregard any contribution to $I_{D}$ from secondary production on G1. Equation (1) describes that $I_{\mathrm{G} 2}$ is made up of the direct impact of dust plus a contribution $I_{S}$ due to secondary charge production. Havnes and Næsheim (2007) found that the net value of $I_{S}$ on the grid they impact is positive. This means that the dust which impacts at high $\theta_{i}$ and fragments, will rub off electrons. This behaviour is also observed in laboratory experiments where fresh surfaces exposed to impacting and fragmenting ice particles, initially gave off negative charges to the ice fragments. After some minutes, this changed and the emission of positive particles dominated (Tomsic, 2001). The current $I_{\mathrm{DC}}$ is made up of the fraction $\left(1-\sigma_{2}\right)$ of $I_{D}$, which is not captured by $\mathrm{G} 2$, plus the secondary charges from $\mathrm{G} 2$ which now gives a contribution to DC an opposite polarity to that on G2. Direct impacts on the bottom plate is not expected to lead to any secondary production, since the impact angle is small and the relative velocity is comparatively low (Dalmann et al., 1977). The secondary production on $\mathrm{G} 2$ will be $\sim 4$ times larger than on G1 and the fragments produced on $\mathrm{G} 2$ are much more likely to reach the bottom plate both because of a $\sim 3$ times smaller air gap than between G1 and G2 and also because the electric field between G2 and DC will accelerate negative fragments toward the bottom plate.

From Eqs. (1) and (2) it follows that

$I_{D}=I_{\mathrm{G} 2}+I_{\mathrm{DC}}$ 

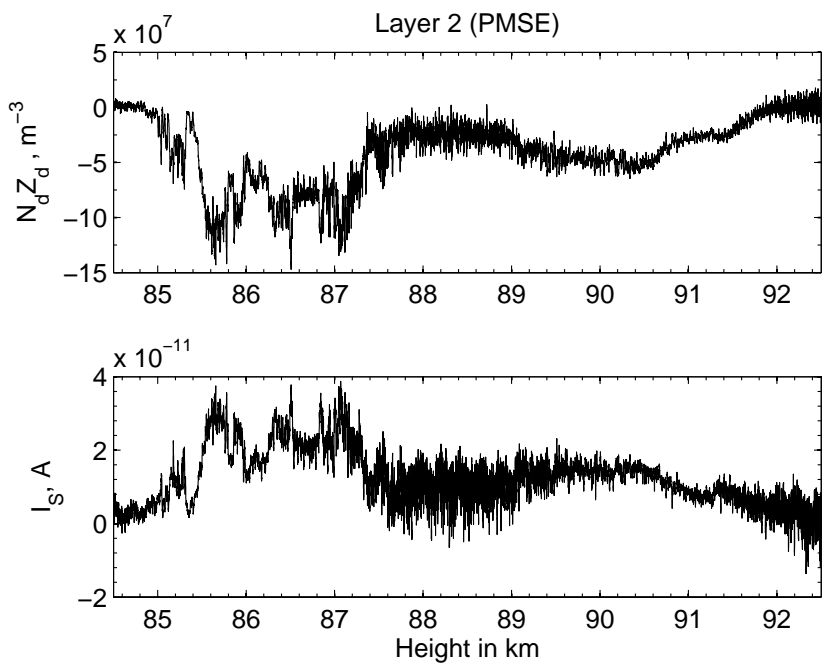

Fig. 8. As for Fig. 7 but for layer 2 (PMSE).

$I_{S}=\left(1-\sigma_{2}\right) I_{\mathrm{G} 2}-\sigma_{2} I_{\mathrm{DC}}$

In Fig. 7, we show in the upper panel the dust charge number density, $N_{d} Z_{d}$ found from Eqs. (3) and (4), and in the lower panel the secondary currents $I_{S}$ for layer 1 . In Fig. 8, we show the same but now for layer 2 . In both cases, $I_{S}$ roughly reflects the variations in dust charge density. This indicates that the secondary charge production per impacting dust particle does not vary dramatically throughout the two layers.

The dust charge density $N_{d} Z_{d}$, shown in Fig. 7 for the NLC/PMSE layer, is considerable and the maximum density of $N_{d} Z_{d} \sim-3.5 \times 10^{9} \mathrm{~m}^{-3}$ is similar to the maximum values measured by DUSTY 1 (ECT-02) for a non-visual PMSE layer (Havnes et al., 1996). For the DUSTY 1 flight these large values of $N_{d} Z_{d}$ coincided with the two deep electron bite-outs each $\sim 0.5 \mathrm{~km}$ wide and we find it likely that the large values of $N_{d} Z_{d}$ in the NLC/PMSE layer must cause one or more electron bite-outs within this layer also. The values of $N_{d} Z_{d}$, for the upper PMSE layer shown in Fig. 8, are low throughout the whole layer and we find it unlikely that values of $\left|N_{d} Z d\right| \lesssim 10^{8} \mathrm{~m}^{-3}$ will give rise to any electron bite-outs in this layer where the electron density most likely is close to two orders of magnitude higher. The rocket did not fly through any of the radar beams of the Alwin MST radar and a direct comparison of the $N_{d} Z_{d}$ height profile with the radar PMSE profile is not possible. It is, however, likely that a PMSE was present along the rocket path in much of the height region of Fig. 8. The vertical Alwin beam observed a PMSE layer extending from $\sim 86.2$ to $89.5 \mathrm{~km}$ during launch. In its upper parts from $\sim 88$ to $89.5 \mathrm{~km}$ a consistent weak PMSE's of $\sim 10-15 \mathrm{~dB}$ above the background was present for more than $10 \mathrm{~min}$ before launch to more than $30 \mathrm{~min}$ after launch. In this region, the dust charge number density was of the order of $N_{d} Z_{d} \sim-5 \times 10^{7} \mathrm{~m}^{-3}$ only. This confirms earlier findings that also very low dust densities with
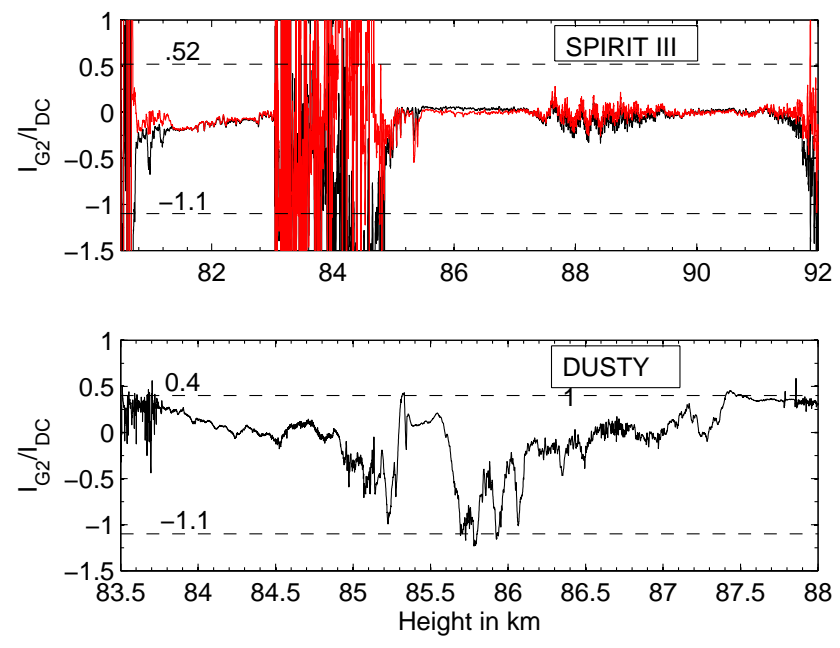

Fig. 9. In the top panel, we show the ratio $R_{S}=I_{\mathrm{G} 2} / I_{\mathrm{DC}}$ for both layer 1 and layer 2 . The very noisy results are from where there is no detectable dust layer so the ratio is just of noise. The red curve is for Fourier representation of the correction for the coning induced background contribution, while the black is for a sinus representation of the correction. We have shown the theoretical limits of $R_{S}$. In the lower panel, we show, only for comparison, the results for $R_{S}$ for the flight DUSTY 1 (Havnes and Næsheim 2007).

$N_{d} Z_{d} \ll n_{e}$ can give rise to PMSE (Havnes et al., 2001a, b; Rapp and Lübken, 2004). The present results indicate that a dust charge density of only one percent, or possibly less, of the electron density may be sufficient to produce a PMSE since the electron density at this height region is most likely $n_{e} \gtrsim 5 \times 10^{9} \mathrm{~m}^{-3}$.

\section{Secondary charge production in the NLC/PMSE and PMSE layer}

The secondary charge currents $I_{S}$, as produced on grid 2 as given by Eq. (5), are shown in Figs. 7 and 8. The importance of the secondary charge production can be seen from the ratio

$$
R_{S}=\frac{I_{\mathrm{G} 2}}{I_{\mathrm{DC}}}=\frac{\sigma_{2} I_{D}+I_{S}}{\left(1-\sigma_{2}\right) I_{D}-I_{S}}
$$

Havnes and Næsheim (2007) showed that for the DUSTY 1 launch (Havnes et al., 1996), $R_{S}$ have all the values within the limits of $R_{S}$ which were $R_{S} \approx \sigma_{2} /\left(1-\sigma_{2}\right) \approx 0.3$ for $I_{S} \rightarrow 0$ and $R_{S} \approx-1$ for $I_{S} \gg I_{D}$. For the ESPRIT dust probe the corresponding limits are $0.47>R_{S}>-1$. In fact, a correction for the slightly higher shadowing of the bottom plate than for G2 at a coning $\gamma \sim 13^{\circ}$, the limits will change to $0.42>R_{S}$ (DUSTY) $>-1.1$ and $0.52>R_{S}(\mathrm{EDD})>-1.1$. In Fig. 9, we show the ratio $R_{S}=I_{\mathrm{G} 2} / I_{\mathrm{DC}}$ for ESPRIT in the upper panel, and for DUSTY 1 in the lower one. The result, when $I_{\mathrm{G} 2}$ has been corrected by a Fourier-approximation to the background current, is shown as a red line, while the correction by a sine-curve is shown as a black line. $I_{\mathrm{DC}}$ was 


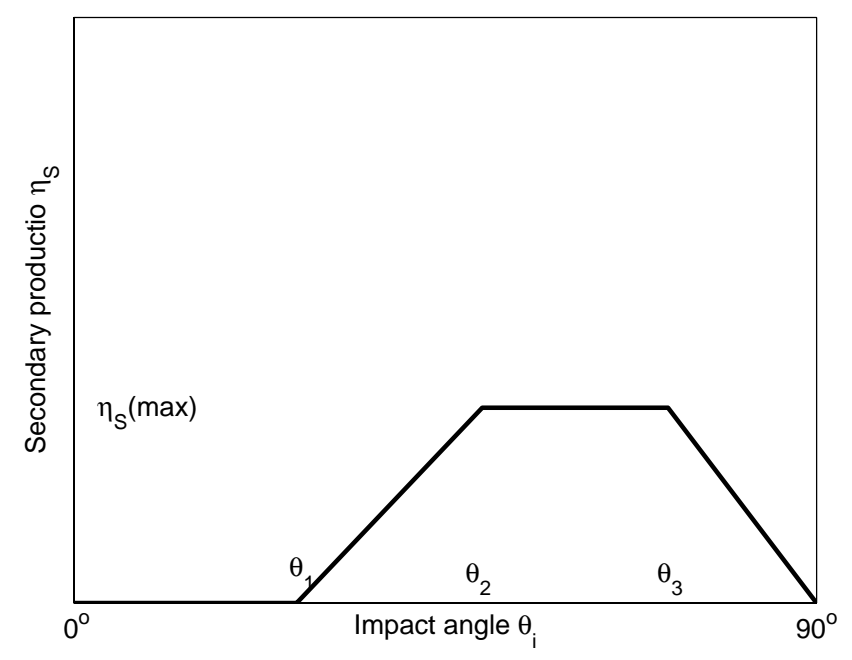

Fig. 10. A schematic representation of the secondary production of charge as a function of impact angle $\theta_{i}$.

only corrected by a sine-curve approximation to the background current. The noisy regions at the upper and lower height regions in both panels and between $\sim 83$ to $84.8 \mathrm{~km}$ for ESPRIT are from regions where both $I_{\mathrm{G} 2}$ and $I_{\mathrm{DC}}$ approach zero so the ratio is just due to noise. The values close to the edges of layer 1 and 2 for ESPRIT are uncertain because the uncertainties in the correction for the background coning-induced current to both $I_{\mathrm{G} 2}$ and $I_{\mathrm{DC}}$.

It is clear from Fig. 9 that a considerable amount of secondary charge production is present, in spite of the small surface of the side edges of the Grid 2 wires where impacts at sufficient high impact angles can occur. If no secondary impacts occur, $R_{S}$ should approach the upper limit. As we see, the ratio $R_{S}$ is not near this value in either of the two layers. In layer 1 , where both currents $I_{\mathrm{G} 2}$ and $I_{\mathrm{DC}}$ are well above the background current, except at the edges, the secondary current is in fact dominant on G2, since this current is positive and $R_{S}<0$. In layer 2 the value of $I_{\mathrm{G} 2}$ is always low which means that the secondary production on G2 approximately balances the direct current $\sigma_{2} I_{D}$ to it. For this to happen, we need a considerable secondary production on the small area at the sides of the grid wires which can produce secondary currents at dust impacts. A critical parameter in this context is the dependence of secondary production $\eta_{s}\left(\theta_{i}\right)$ on the impact angle $\theta_{i}$. Based on laboratory experiments (Tomsic, 2001), the dependence of secondary production on $\theta_{i}$ is most likely one where there is no secondary production for low $\theta_{i}<\theta_{1}$, an increasing production for $\theta_{1}<\theta_{i}<\theta_{2}$, a maximum production between $\theta_{2}$ and $\theta_{3}$ and a decreasing production from $\theta_{i}=\theta_{3}$ to $\eta_{s}=0$ at $\theta_{i}=90^{\circ}$, as shown in Fig. 10. The value of $\eta_{s}\left(\theta_{i}\right)$ corresponds to the number of electrons which are carried away by one impacting dust particle. Havnes and Næsheim (2007) found that in order to explain the dust and secondary production obser-

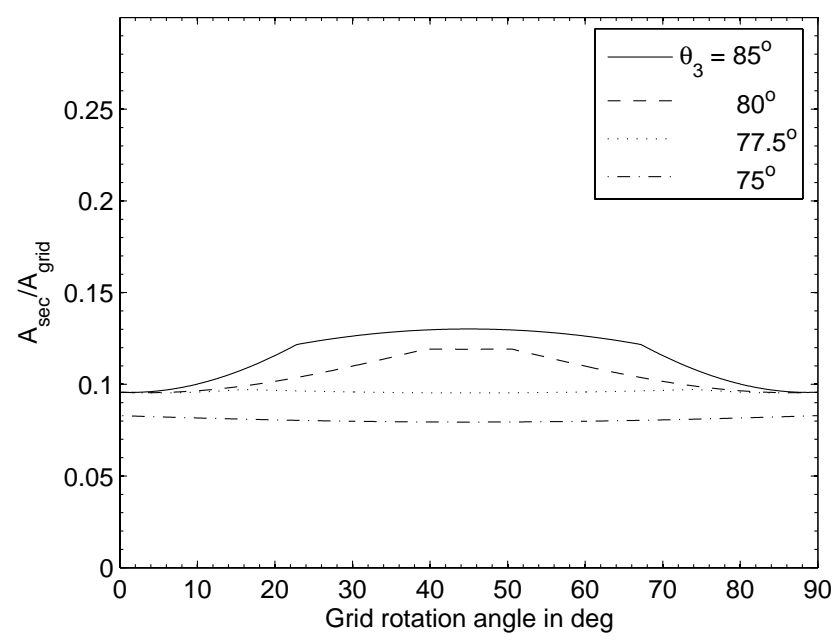

Fig. 11. The variation of effective area for secondary charge production as a function of payload rotation angle for different values of $\theta_{3}$ (Fig. 10). The value of $\eta_{S}(\max )$ used here is 1 .

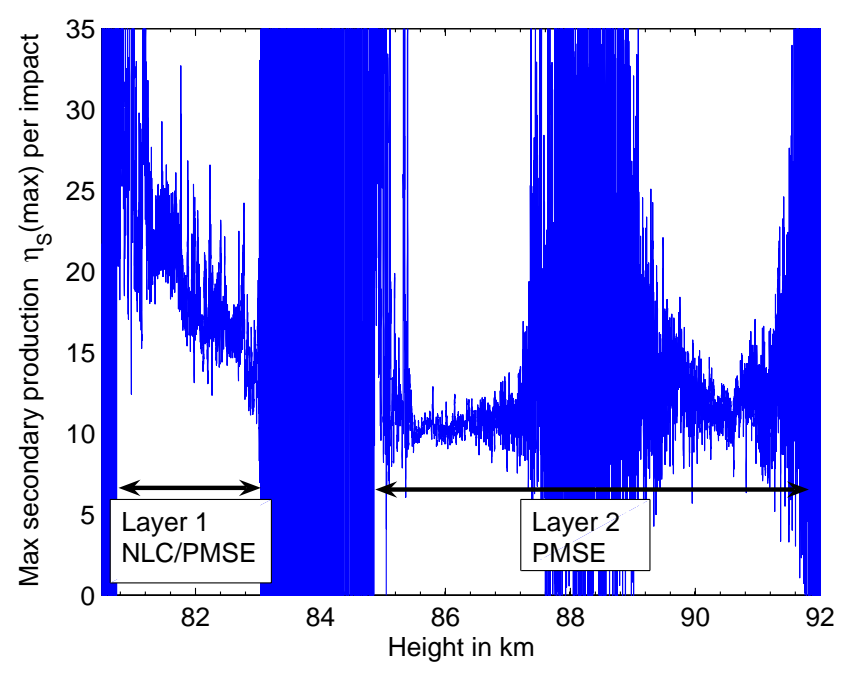

Fig. 12. The computed $\eta_{S}(\max )$ for the two dust layers. We show here the results for the sinus-correction for the background variations. The Fourier representation gives only small changes to the results in the two layers.

vations from DUSTY 2 (Havnes et al., 1996), the onset of secondary production had to be at an angle $\theta_{1} \sim 20-35^{\circ}$ as compared to the $\theta_{1} \sim 45^{\circ}$ observed in experiments for pure ice particles (Tomsic, 2001). Also, they found that a large secondary production should already result at impact angles as low as $\theta_{i} \sim 55^{\circ}$, while Tomsic (2001) finds $\theta_{2} \sim 70^{\circ}$ for ice particles. The reason for the comparatively high value of the impact angles found in the experiments, compared to those required for mesospheric dust particles, is most likely that the pure ice particles in the experiments will totally sublimate at lower impact angles (Tomsic, 2001). On the other hand, mesospheric ice particles may contain many small 
meteoric particles (Rosinski and Snow, 1961; Hunten et al., 1980; Megner et al., 2006) which probably do not sublimate. Havnes and Næsheim (2007) suggested that mesospheric dust particles fragment during impact and that much or all of the ice within which the meteoric smoke particles are embedded, sublimates, while the meteoric smoke particles carry away charge from the surface where the impact takes place. The secondary charging effect of impacting meteoric smoke particles may have been observed in rocket experiments during winter conditions (Amyx et al., 2008). On grid 2 of EDD the secondary production can only take place on one of the side edges of the square wire profiles (Fig. 1) and the impact angles will vary between $90-\gamma \leq \theta_{i} \leq 90^{\circ}$ as the payload rotates. The coning angle when the payload passes through the NLC and PMSE layers is $\gamma \sim 13^{\circ}$. The impact angle on the two side-edges $x$ and $y$ in each square of the grid, which is exposed to impacts at high $\theta_{i}$, have impact angles given by $\cos \theta_{i x}=\sin \gamma \cdot \cos \varphi(t)$ and $\cos \theta_{i y}=\sin \gamma \cdot \sin \varphi(t)$ where $\varphi(t)$ is the rotation angle of the payload. The total effective grid area $A_{\text {grid }}$ for direct impacts will vary slightly during payload rotation because a varying amount of side-edge of the grid wire will be exposed to the incoming dust. Because of the comparatively small coning, the exposed sideedge area will be in the region of $10-15 \%$ of the upper edge area of the grid. Secondary charges should only be produced on the exposed side-edges where the particles hit with impact angles varying between $90^{\circ}-\gamma=77^{\circ}$ and $90^{\circ}$ as the payload rotates. The effective area $A_{\text {sec }}$ of a side-edge for secondary charge production is equal to the area of the side-edge normal to the ram direction, multiplied with the value of secondary production (Fig. 10) $\eta_{s}$ at the relevant impact angle. Figure 11 shows the ratio $A_{\mathrm{sec}} / A_{\text {grid }}$ between the effective area for secondary production and the total grid area for different values of $\theta_{3}$ with $\gamma=13^{\circ}$. The effective area $A_{\mathrm{sec}}$ for secondary production is calculated here by use of a secondary production $\eta_{s}\left(\theta_{i}\right)$ which has a maximum value $\eta_{s}(\max )=1$. We later calculate the true values of $\eta_{s}(\max )$ in Fig. 10 which is required to reproduce the observed secondary production. We see that for $\theta_{3}<90-\gamma \sim 77^{\circ}$ there is a negligible variation in the ratio while a variation starts to develop when $\theta_{3}>90-\gamma=77^{\circ}$. This variation will be at a frequency of 4 times the payload rotation frequency. We see, however, from the frequency analysis in Fig. 6, that there is no or a very weak contribution at $4 f_{R}$ in both the layers (panels a and b) which indicate that the value of $\theta_{3}$ should be below $\sim 80^{\circ}$ and that the secondary production falls off at higher $\theta_{i}$ to become 0 at $\theta_{i}=90^{\circ}$. Adopting the values $\theta_{3}=75^{\circ}$ which was used by Havnes and Næsheim (2007), we have the ratio $A_{\text {sec }} / A_{\text {grid }} \sim 0.082$ at all rotation angles. This enables us to compute the value $\eta_{s}(\max )$, the number of electrons rubbed off by one impacting dust particle, which is needed to explain the observed secondary current $I_{S}$. From Eq. (3), we know that the total flux of dust particles between grid 1 and 2 is $I_{D} / Z_{d}$. The fraction of the total flux which impacts on the edges of the grid wires to produce secondary charges is
$\approx\left(A_{\text {sec }} / A_{\text {grid }}\right) \cdot \sigma_{2} \approx 0.023$. Each of the impacts will produce $\eta_{s}(\max )$ secondary electrons so we have

$I_{S}=\eta_{s}(\max ) \cdot 0.023 \cdot \frac{I_{D}}{Z_{d}}$.

This enables us to find $\eta_{s}$ (max) by the use of Eqs. (4) and (5). We have plotted the results in Fig. 12 with $Z_{d}=-1$. For layer 1 , which most likely consists of fairly large NLC dust particles of radius $r_{d} \approx 50 \pm 20 \mathrm{~nm}$ (von Cossart et al., 1999; Eremenko et al., 2005), the real $Z_{d}$ should be higher than -1 , a likely charge range could be $Z_{d}=-2$ to -4 . This shows that for this NLC/PMSE layer, the production factor $\eta_{s}(\max )$ may well be from 50 to 100 . This high number is in the range for $\eta_{s}(\max )$ which was also found by Havnes and Næsheim (2007). For layer 2 we have no direct information on the dust sizes. This layer most likely consists of smaller particles, since it was not detected by lidars. Their charges will be close to $Z_{d} \sim-1$ leading to values of $\eta_{s}(\max )$ of $\sim 10-15$.

\section{Discussion}

The dust observations by the dust probe EDD on the ESPRIT payload launched as SPIRIT III confirmed that charged dust particles are present in both visual dust layers (NLC/PMSE) and in non-visual dust layers (PMSE). The maximum dust charge densities in layer 1 (Fig. 7), the NLC layer, is $N_{d} Z_{d} \sim-3.5 \times 10^{9} \mathrm{~m}^{-3}$ which is close to the maximum values which have been found in earlier rocket flights (Havnes et al., 1996; Smiley et al., 2006). The dust charge density is expected to be smaller or, in the case of a strong electron bite-out where most of the electrons are captured by the dust particles, comparable to the electron density just outside the clouds (Havnes et al., 2001a; Rapp et al., 2003). This means that at the NLC of $\sim 82 \mathrm{~km}$ for ESPRIT, the electron density without dust should be $n_{e} \gtrsim 4 \times 10^{9} \mathrm{~m}^{-3}$. In the much higher layer 2 between $\sim 85$ to $91.5 \mathrm{~km}$ height, where $N_{d} Z_{d}$ is low, the electron density should not be much affected, and we expect that the electron density must be appreciably higher at least by a factor of 2 . This makes it probable that the observed dust charge densities of $N_{d} Z_{d} \lesssim-10^{8} \mathrm{~m}^{-3}$ shown in Fig. 8 , not only confirms that very little dust, compared to the electron density, is required for PMSE to be formed, but that a ratio as low as $P=\left|N_{d} Z_{d} / n_{e}\right| \lesssim 0.01$ can be sufficient. This is an observed record low $P$ for PMSE conditions and confirms the results of Havnes et al. (2001a, b) (see also Rapp and Lübken, 2004) that earlier beliefs that $P \sim 1$ was required for PMSE to form, was not correct.

A very important result of the SPIRIT III dust detector is that it confirms that secondary charge production by impacting dust particles must always be considered. If dust can impact on probe or payload surfaces with an impact angle in the range $20^{\circ}-35^{\circ} \lesssim \theta_{i}$ it is likely that secondary charge effects will have an influence on the payload charging and probe currents. In the present dust detector EDD the grid 
wires were constructed to reduce the secondary production but they were still present. In DUSTY (Havnes et al., 1996) the area of grid 2 (see Fig. 1) producing secondary charges is about $30 \%$ of the total grid area while for the EDD it is $\sim 10 \%$. The non-negligible secondary production, in spite of the small part of the grid surface available for this case, shows that in the NLC region the impacting dust particles can each rub off a maximum number of negative charges in the range 50 to 100. Havnes and Næsheim (2007) also found that this was required to explain the large positive currents on the front grid of their dust probe in the DUSTY 2 (ECT-07) flight. This confirmation, that the secondary production is very much higher for mesospheric dust particles than for pure ice particles in experiments, gives support to the model for the mesospheric dust proposed by Havnes and Næsheim (2007). We, therefore, find it now even more probable that mesospheric dust contains a considerable number of meteoric nanometer small smoke particles and that they are the ones which rub off charges from the surfaces which are impacted, while the water in the mesospheric dust particles mainly sublimates. However, it may be a problem for the model that the effectivity, of the fragments of the mesospheric dust particles, is found to be so much higher than pure ice particles in rubbing off electrons. According to the estimates by Havnes and Næsheim (2007), the effectivity must be at least one and possibly several orders of magnitude larger than similar sized ice particles of which typically only one out of 1000 rub off an electron during impact. A considerable part of the difference can be explained because apparently all of the ice particles evaporate if their sizes are smaller than $\sim 6 \mathrm{~nm}$, and many larger also (Tomsic, 2001), while the smoke particles are much less likely to sublimate. We do not know if a mixing of meteoric metals in atomic form with the condensed water ice in the mesospheric dust (Havnes et al., 1990; Lübken and Hoffner, 2004; Plane et al., 2004) also can increase the probability for secondary impact effects. However, it seems clear that a correct model for the mesospheric dust particles must be quite different from that of a pure ice particle, condensed around a small meteoric smoke particle.

Acknowledgements. Topical Editor U.-P. Hoppe thanks two anonymous referees for their help in evaluating this paper.

\section{References}

Amyx, K., Sternovsky, Z., Knappmiller, S., Robertson, S., Horányi, $\mathrm{M}$, and Gumbel, J.: In-situ measurement of smoke particles in the wintertime polar mesosphere between 80 and $85 \mathrm{~km}$ altitude, J. Atmos. Sol. Terr. Phys., 70, 61-70, 2008.

Andersson, P. U. and Pettersson, J. B. C.: Ionization of water clusters by collisions with graphite surfaces, Z. Phys. D, 41, 57-62, 1997.

Barjatya, A. and Swenson, C. M.: Observations of triboelectric charging effects on Langmuir-type probes in dusty plasma, J. Geophys. Res., 111, A10, doi:10.1029/2006JA011806, 2006.
Björn, L. G. and Arnold, F.: Mass spectrometric detection of precondensation nuclei at the arctic summer mesopause, Geophys. Res. Lett., 8, 1167-1170, 1981.

Cho, J. Y. N. and Röttger, J.: An updated review of polar mesosphere summer echoes: Observations, theory, and their relationship to noctilucent clouds and subvisible aerosols, J. Geophys. Res., 102, 2001-2020, 1997.

Dalmann, B.-K., Grün, E., Kissel, J., and Dietzel, H.: The ioncomposition of the plasma produced by impacts of fast dust particles, Plan. Space Sci., 25, 135-147, 1977.

Ecklund, W. L. and Balsley, B. B.: Long-term observations of the Arctic mesosphere with the MST radar at Poker Flat, Alaska, J. Geophys. Res., 86, 7775-7780, 1981.

Edwards, P. S., Philbrick, C. R., Surdal, L. H., Johnsen, M. G., Latteck, R., and Baumgarten, G.: The ESPRIT rocket payload launch: Initial comparison with ground based atmospheric measurements, Proc. 18th ESA Symposium on European Rocket and Balloon Programmes and Related Research, Visby, Sweden, 3-7 June 2007 (ESA SP-647), 139-144, 2007.

Eremenko, M. N., Petelina, S. V., Zasetsky, A. Y., Karlsson, B., Rinsland, C. P., Llewellyn, E. J., and Sloan, J. J.: Shape and composition of PMC particles derived from satellite remote sensing measurements, Geophys. Res. Lett., 32, L16S06, doi:10.1029/2005GL023013, 2005.

Escobar, A. C., Bilén, S. G., and Philbrick, C. R.: Preliminary Langmuir probe analysis from ESPRIT, Proc. 18th ESA Symposium on European Rocket and Balloon Programmes and Related Research, Visby, Sweden, 3-7 June 2007 (ESA SP-647), 263-268, 2007.

Gadsden, M. and Schröder, W.: Noctilucent Clouds, Physics and chemistry in space 18, Springer-Verlag, 1989.

Gridin, V. V., Gebhardt, C. R., Tomsic, A., Schechter, I., Schröder, H., and Kompa, K. L.: Surface impact induced fragmentation and charging of neat and mixed clusters of SO2 and H2O, Int. J. Mass Spectrom., 232, 1-7, 2004.

Gumbel, J. and Witt, G.: In situ measurements of the vertical structure of a noctilucent cloud, Geophys. Res. Lett., 25, 493-496, 1998.

Hallgren, P. S., Schmalberger, D. C., and Hemenway, C. L.: Noctilucent cloud sampling by multi-experiment payload, Space Res., XII, 1105-1112, 1973.

Havnes, O. and Kassa, M.: On the sizes and observable effects of dust particles i PMWE, J. Geophys. Res., accepted, doi:2008JD011276, 2009.

Havnes, O. and Næsheim, L. I.: On the secondary charging effects and structure of mesospheric dust particles impacting on rocket probes, Ann. Geophys., 25, 623-637, 2007, http://www.ann-geophys.net/25/623/2007/.

Havnes, O., de Angelis, U., Bingham, R., Goertz, C. K., Morfill, G. E., and Tsytovich, V.: On the role of dust in the summer mesopause, J. Atmos. Terr. Phys., 52, 637-643, 1990.

Havnes, O., Trøim, J., Blix, T., Mortensen, W., Næsheim, L. I., Thrane, E., and Tønnesen, T.: First detection of charged dust particles in the Earth's mesosphere, J. Geophys. Res., 101, 10839$10847,1996$.

Havnes, O., Brattli, A., Aslaksen, T., Singer, W., Latteck, R., Blix, T., Thrane, E., and Trøim, J.: First common volume observations of layered plasma structures and polar mesospheric summer echoes by rocket and radar, Geophys. Res. Lett., 28, 1419-1422, 
2001a.

Havnes, O., Aslaksen, T., and Brattli, A.: Charged dust in the Earth's middle atmosphere, Phys. Scripta, T89, 133-137, $2001 \mathrm{~b}$.

Hedin, J., Gumbel, J., and Rapp, M.: On the efficiency of rocketborne particle detection in the mesosphere, Atmos. Chem. Phys., 7, 3701-3711, 2007,

http://www.atmos-chem-phys.net/7/3701/2007/.

Hedin, J., Gumbel, J., Rapp, M., and Waldemarsson, T.: On the efficiency of rocket-borne particle detection in the mesosphere, 36th COSPAR Scientific Assembly, p. 3098, Beijing, China, 1623 July 2006.

Horányi, M., Gumbel, J., Witt, G., and Robertson, S.: Simulation of rocket-borne particle measurements in the mesosphere, Geophys. Res. Lett., 26, 1537-1540, 1999.

Hunten, D. M., Turco, R. P., and Toon, O. B.: Smoke and dust particles of meteoric origin in the mesosphere and stratosphere, J. Atmos. Sci., 37, 1342-1357, 1980.

Kopp, E., Bertin, F., Björn,L.G., Dickinson, P. H. G., Philbrick, C. R., and Witt, G.: The CAMP campaign 1982, Proc. 7th ESA Symposium on European Rocket and Balloon Programmes and Related Research, Loen, Norway, 5-11 May 1985, (ESA SP229), 117-123, 1985.

Latteck, R., Singer, W., and Bardey, H.: The ALWIN MST Radar Technical Design and Performance, Proceedings 14th ESA Symposium on European Rocket and Balloon Programmes and Related Research, Potsdam, Germany, 31 May-3 June 1999, ESA SP-437, pp. 179-184, 1999.

Lübken, F. J. and Hoffner, J.: Experimental evidence for ice particle interaction with metal atoms at the high latitude summer mesopause region, Geophys. Res. Lett., 31, L08103, doi:10.1029/2004GL019586, 2004.

Megner, L., Rapp, M., and Gumbel, J.: Distribution of meteoric smoke - sensitivity to microphysical properties and atmospheric conditions, Atmos. Chem. Phys., 6, 4415-4426, 2006,

http://www.atmos-chem-phys.net/6/4415/2006/.

Mitchell, J. D., Croskey, C. L., and Goldberg, R. A., Evidence for charged aerosols and associated meter-scale structure in identified PMSE/NLC regions, Geophys. Res. Lett., 28, 1423-1426, 2001.

Pedersen, A., Trøim, J., and Kane, J.: Rocket measurement showing removal of electrons above the mesopause in summer at high latitudes, Planet. Space Sci., 18, 945-947, 1969.

Philbrick, C. R., Bilén, S. G., Wheeler, T. F., and Thrane, E. V.: Educational advantages afforded by the ESPRIT project, Proc. 18th ESA Symposium on European Rocket and Balloon Programmes and Related Research, Visby, Sweden, 3-7 June 2007 (ESA SP647), 257-262, 2007.

Plane, J. M. C.: Atmospheric chemistry of meteoric metals, Chem. Rev., 103, 4963-4984, 2003.

Plane, J. M. C., Murray, B. J., Chu, X. Z., and Gardner, C. S.: Removal of meteoric iron on polar mesospheric clouds, Science, 304, 426-428, 2004.

Rapp, M. and Lübken, F.-J.: Polar mesosphere summer echoes (PMSE): Review of observations and current understanding, Atmos. Chem. Phys., 4, 2601-2633, 2004, http://www.atmos-chem-phys.net/4/2601/2004/.
Rapp, M., Lübken, F.-J., and Blix, T. A.: Small scale density variations of electrons and charged particles in the vicinity of polar mesosphere summer echoes, Atmos. Chem. Phys., 3, 13991407, 2003, http://www.atmos-chem-phys.net/3/1399/2003/.

Rapp, M., Hedin, J., Strelnikova, I., Friedrich, M., Gumbel, J., and Lübken F.-J.: Observations of positively charged nanoparticles in the nighttime polar mesosphere, Geophys. Res. Lett., 32, L23821, doi:10.1029/2005GL024676, 2005.

Reichard, G., Cochrane, C., and Fortin, W.: ESPRIT sun sensor: Results and future use, Proc. 18th ESA Symposium on European Rocket and Balloon Programmes and Related Research, Visby, Sweden, 3-7 June 2007 (ESA SP-647), 2007.

Rosinski, J. and Snow, R. H.: Secondary particulate matter from meteor vapors, J. Meteorol., 18, 736-745, 1961.

Schulte, P. and Arnold, F.: Detection of upper atmospheric negatively charged microclusters by a rocket-borne massspectrometer, Geophys. Res. Lett., 19, 2297-2300, 1992.

Smiley, B., Rapp, M., Blix, T. A., Robertson, S., Horányi, M., Latteck, R., and Fiedler, J.: Charge and size distribution of mesospheric aerosol particles measured inside NLC and PMSE during MIDAS MaCWAVE 2002, J. Atmos. Solar-Terr. Phys., 68, 114-123, 2006.

Thomas, G. E.: Mesospheric clouds and the physics of the mesopause region, Rev. Geophys., 29, 553-575, 1991.

Tomsic, A.: Collisions between water clusters and surfaces, Dr. filos. thesis, Gothenburg University, Gothenburg, 2001.

Ulwick, J. C., Baker, K. D., Kelley, M. C., Balsley, B. B., and Ecklund, W. L.: Comparison of simultaneous MST radar and electron density probe measurements during STATE, J. Geophys. Res., 93, 6989-7000, 1988.

von Cossart, G., Fiedler, J., and von Zahn, U.: Size distributions of NLC particles as determined from 3-color observations of NLC by ground-based lidar, Geophys. Res. Lett., 26, 1513-1516, 1999.

von Zahn, U. and Bremer, J.: Simultaneous and common-volume observations of noctilucent clouds and polar mesosphere summer echoes, Geophys. Res. Lett., 26, 1521-1524, 1999.

von Zahn, U., Thrane, E. V., and Skatteboe, R.: The ALOMAR facility: Status and outlook, in Proceedings 12th ESA Symposium on European Rocket and Balloon Programmes and Related Research, Lillehammer, Norway, (ESA SP,) pp. 379-385, 1995.

Vostrikov, A. A., Zadorozhny, A. M., Dubov, D. Y., Witt, G., Kazakova, I. V., Bragin, O. A., Kazakov, V. G., Kikhtenko, V. N., and Tyutin, A. A.: Ioozation of water clusters by collision with surface, Z. Phys. D, 40(1-4), 542-545, 1997.

Weingartner, J. C. and Draine, B. T.: Electron-ion recombination on grains and poycyclic aromatic hydrocarbons, Astrophys. J., 563, 842-852, 2001.

Zadorozhny, A. M., Tyutin, A. A., Witt, G., Wilhelm, N., Walchli, U., Cho, J. Y. N., and Swartz, W. E.: Electric field measurements in the vicinity of noctilucent clouds and PMSE, Geophys. Res. Lett., 20, 2299-2302, 1993. 